\title{
Tumor necrosis factor receptor-associated periodic syndrome (TRAPS) in a Dutch family: evidence for a TNFRSF1A mutation with reduced penetrance
}

\author{
Ebun Aganna ${ }^{1}$, Ivona Aksentijevich², Graham A Hitman ${ }^{1}$, Daniel L Kastner ${ }^{2}$, \\ Andy IM Hoepelman ${ }^{3}$, Fokke D Posma ${ }^{4}$, Egbert JK Zweers ${ }^{5}$ and Michael F McDermott ${ }^{1}$ \\ ${ }^{1}$ Unit of Molecular Medicine, Department of Diabetes and Metabolic Medicine, St Bartholomew's and the Royal \\ London School of Medicine and Dentistry, Queen Mary and Westfield College, London, UK; ${ }^{2}$ Arthritis and \\ Rheumatism Branch, National Institute of Arthritis and Musculoskeletal and Skin Diseases, Bethesda, MD, USA; \\ ${ }^{3}$ Department of Medicine, Division of Infectious Diseases and AIDS and Eijkman-W inkler Institute, University \\ Medical Center Utrecht; ${ }^{4}$ Department of Clinical Chemistry, Twenteborg Hospital, Almelo; ${ }^{5}$ Department of M edicine, \\ University Medical Center Utrecht, The N etherlands
}

Mutations of the tumor necrosis factor receptor 1 (TNFRSF1A) gene underly susceptibility to a subset of autosomal dominant recurrent fevers (ADRFs). We report on a two-generation six-member Dutch family in which a novel R92P mutation and reduced plasma TNFRSF1A levels were found in all the children, including two who are unaffected. However, only the daughter proband and father exhibited a typical TNF-receptor associated periodic syndrome (TRAPS) phenotype. PCR-RFLP analysis revealed that the mutation was not present in $\mathbf{1 2 0}$ control chromosomes from unaffected Dutch individuals. As this R92P mutation is present in two unaffected carriers it appears to be less penetrant than previously reported TNFRSF1A mutations involving cysteine residues in the extracellular domains. European Journal of Human Genetics (2001) 9, 63-66.

Keywords: TNFRSF1A mutation; TRAPS; penetrance; plasma TNFRSF1A levels

\section{Introduction}

Autosomal dominant and recessive periodic fevers comprise a group of disorders characterised by fevers, peritonitis, pleurisy, arthritis, skin rashes, and/or conjunctivitis. These attacks are separated by symptom-free intervals and some patients devel op AA amyloidosis. The discovery of mutations of the tumor necrosis factor receptor 1 (TNFRSF1A or tumor necrosis factor receptor superfamily $1 A$ ) gene in a subset of autosomal dominant recurrent fevers (ADRF) has simplified disease classification to some extent. ${ }^{1,2}$

The TNF-Receptor Associated Periodic Syndrome (TRAPS) subsumes a number of older diagnostic entities, including a condition known as familial Hibernian fever $^{3}$ (FHF; OMIM No 142680). The TRAPS phenotype is distinguished by

Correspondence: Dr Michael F McDermott, 5th Floor, Alexandra Wing, The Royal London Hospital, Whitechapel, London, E1 1BB, UK. Tel: +44207377 7000 ext 2259/2269; Fax: +44 20207377 7636. E-mail: M.F.McDermott@mds.qmw.ac.uk

The first two authors contributed equally to this work. Received 29 May 2000; revised 22 August 2000; accepted 30 August 2000 attacks of fever of longer duration, which are mostly resistant to colchicine prophylaxis but partly responsive to steroid therapy. Five of the six TNFRSF1A mutations reported to date disrupt disulfide bonds in extracellular domains. ${ }^{1}$

Familial Mediterranean fever (FMF: OMIM No249100), and hyperimmunoglobulinaemia D (HIDS; OMIM No 260920) are the most common autosomal recessive periodic fevers with FMF being the most prevalent overall condition. ${ }^{4}$ HIDS has been diagnosed in fewer than 150 people and is most commonly found in the Dutch population.

We report on a two-generation Dutch family, where recurrent attacks of fever and abdominal pain appear to segregate as an autosomal dominant trait. We used informative markers from FMF, HIDS and TRAPS loci to study the family. Linkage analysis was unsuitable in this family due to its structure and size, but genotyping data did support a diagnosis of TRAPS and were not consistent with either FMF or HIDS. DNA sequencing of TNFRSF1A as a candidate susceptibility gene revealed a novel R92P mutation that segregates with disease, at reduced penetrance, in this family. 


\section{Methods}

\section{Families and DNA samples}

The family studied includes six living members, two of whom have a clinical phenotype strongly resembling TRAPS (the daughter proband and affected father), and in addition a son aged 27 has mild symptoms suggestive of irritable bowel disease. The Human Experimentation Committee at each participating institution approved the study, and all participants gave informed consent. Clinical details of this family have been described previously. ${ }^{5}$ In summary, the 37-year-old proband daughter became affected at age 24 with recurring abdominal pain at two-month intervals with fevers up to $38.5^{\circ} \mathrm{C}$ but no joint pains or skin rashes. The attacks are of days to weeks duration and are not related to menses. An ultrasound in 1988 showed a swollen right adnex which was surgically removed - there were no abnormal histopathological findings. Her laboratory tests reveal ed an acute phase response with elevated ESR (37-111 mm/h), leukocytosis and elevated CRP at $22.5 \mathrm{mg} / 100 \mathrm{ml}(\mathrm{N}<0.5 \mathrm{mg} / 100 \mathrm{ml})$. Her serum IgD and IgA levels were normal, as were bowel $X$-rays. Serological tests for ANF and anti-dsDNA were negative and there was no evidence of active viral or bacterial infections. Treatment with colchicine produces less frequent and milder attacks. The 69-year-old father developed similar symptoms at 38 years but the attacks are less frequent than in the proband (2-3times per year). He also had coronary bypass operations at 46 and 53 years. Investigations include raised ESR during attacks and normal IgD levels. The father's symptoms also respond to colchicine and anti-inflammatories. A 34-year-old son complains of intermittent abdominal pains but does not have any fevers or arthralgia. Blood was taken from all family members of DNA studies and also from 60 unaffected Dutch individuals who were studied as normal controls.

\section{Microsatellite markers}

TNFRSF1A locus (chromosome12p13) An informative microsatel lite marker (TNFRp55) ${ }^{6}$ from the first intron of the TNFRSF1A gene in addition to the D12S99 and D12S77 flanking markers was used to genotype all family members.

FMF locus (chromosome16p13.3) Two microsatellite markers which tightly flank the MEFV gene, mutated in FMF, were used: D16S3070 (telomeric) and D16S3275 (centromeric) the MEFV locus plus D16S418, about $30 \mathrm{cM}$ centromeric to MEFV.

HIDS locus (chromosome12q24) D12S79 and D1S306 flanking markers were used. ${ }^{7}$

\section{Mutation detection by fluorescent sequencing}

The proband daughter was screened for TNFRSF1A mutations in exons 1-7, as described. ${ }^{1}$ The $5^{\prime}$ untranslated region was also investigated: the complete $900 \mathrm{bp}$ segment was first amplified using two external primers and the product was then sequenced using overlapping internal primers (Amersham, UK).

\section{Restriction endonuclease assays for TNFRSF1A mutations}

The restriction endonuclease assay for the R92P mutation involved the introduction of an enzyme recognition site into the reverse primer by nucleotide substitution. The following primers were used: forward primer $5^{\prime}$ TTACAGAGACACACACTTAGG $3^{\prime}$ and reverse primer $5^{\prime}$ CCTGCAGCCACACACGGTGCCC 3 '. The ' $C$ ' nucleotide in the reverse primer is substituted for the ' $T$ ' nucleotide at position 842 in the published sequence (reference M75865 GenBank) so as to create the Smal restriction enzyme (New England, Biolabs, Herts, UK) recognition sequence. An R92P mutation within the amplified product thus generates a restriction site. $50-100 \mathrm{ng}$ of genomic DNA was amplified in a volume of $15 \mu \mathrm{l}$ in the presence of $1.5 \mathrm{mmol} \mathrm{MgCl}_{2} \mathrm{PCR}$ buffer, 0.06 units of Taq Polymerase (Promega, Southampton, UK), and $5 \mathrm{pmol}$ of each of the appropriate PCR primers. PCR was at $95^{\circ} \mathrm{C}$ for $10 \mathrm{~min}$, with 30 cycles of $95^{\circ} \mathrm{C}$ for $30 \mathrm{~s}, 60^{\circ} \mathrm{C}$ for $30 \mathrm{~s}$, and $72^{\circ} \mathrm{C}$ for $30 \mathrm{~s}$, and the final extension at $72^{\circ} \mathrm{C}$ for $10 \mathrm{~min}$. Amplified products were digested for at least 2 hours with 10 units of Smal and samples loaded on a $4-20 \%$ polyacrylamide gel (Flowgen, Leics, UK). The gel was stained with ethidium bromide and controls included.

\section{Measurement of Soluble TNF Receptors}

Soluble plasma levels of TNFRSF1A (p55) and TNFR2 (p75) were measured by solid-phase ELISA (R\&D Systems, Abingdon, UK) as described. ${ }^{1}$

\section{Results}

\section{Microsatellite markers}

All the children inherited allele 6 of the TNFRp55 marker from the affected father so there was complete segregation of this allele with the low soluble TNFRSF1A levels (Figure1). None of the children were haploidentical for either the FMF or HIDS markers as might be expected for an autosomal recessive disease - in fact the proband daughter and her mildly affected brother do not share haplotypes at either loci (Figure2).

\section{Mutation detection}

Sequencing of the TNFRSF1A gene in the daughter proband revealed a novel $\mathrm{G}$ to $\mathrm{C}$ transversion in exon 4 producing an arginine to proline mutation at residue92 (R92P). The Smal RFLP assay revealed that this mutation was present in all family members except the mother (Figure 1 ) and that it was not found in any of the 60 controls.

\section{Soluble TNFR levels}

Reduced plasma TNFRSFIA levels who found in all the children, including two who are unaffecated (Figure1). The lowest soluble TNFRSF1A levels were observed in the affected proband daughter $(444 \mathrm{pg} / \mathrm{ml})$ and the son with mild symptoms $(517 \mathrm{pg} / \mathrm{ml})$ but low levels were also found in the two unaffected carriers $(547,602 \mathrm{pg} / \mathrm{ml}$ respectively), whereas the 


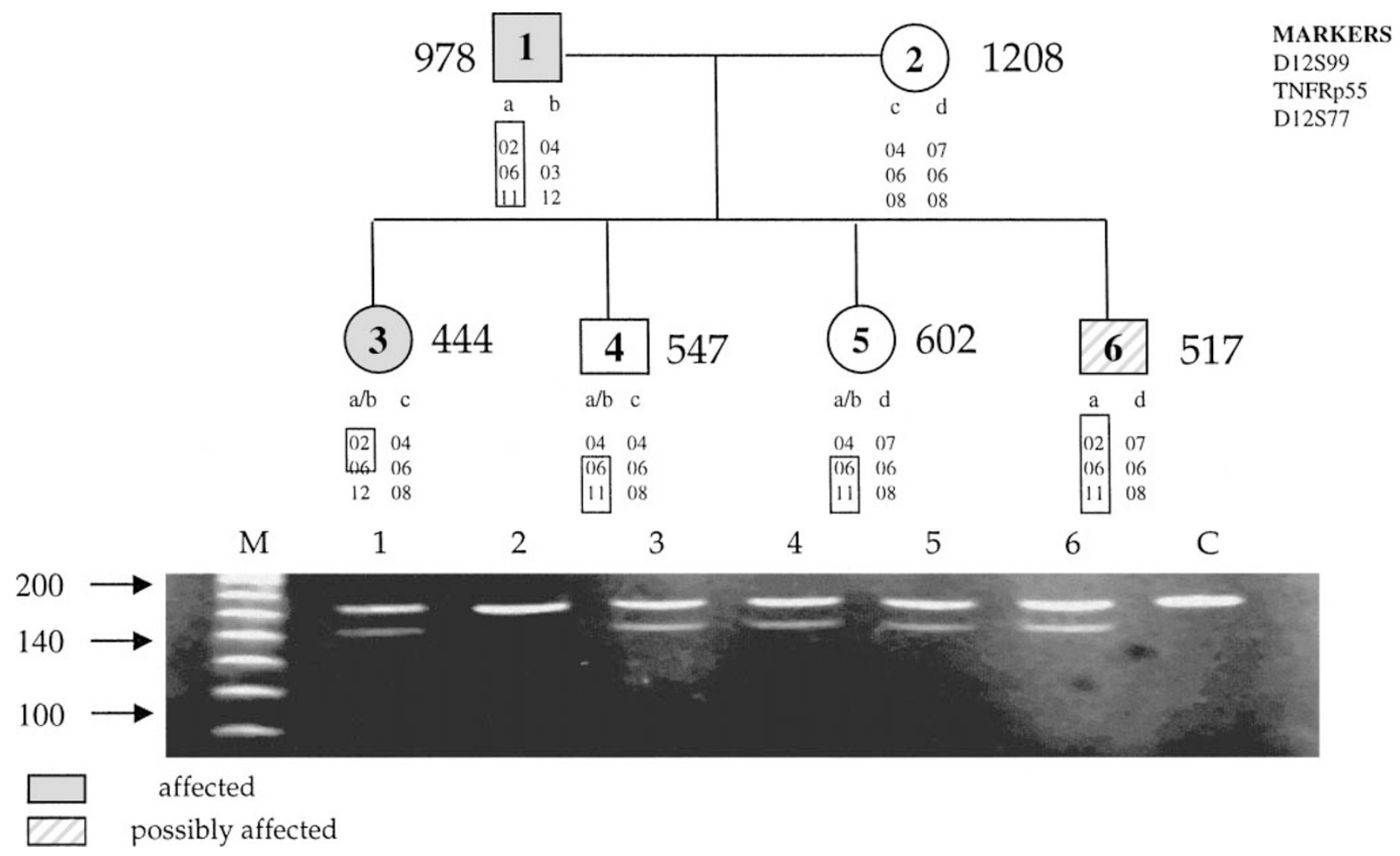

Figure 1 Pedigree of the Dutch family with genotypes of three markers spanning the TNFRSF1A locus on chromosome12p13 and a picture of the Smal RFLP assay for the R92P mutation. Samples were loaded on 16\% polyacrylamide gels (Novex, UK) and stained with ethidium bromide. Positive and negative controls were included in each run. The numbers besides each symbol correspond to soluble TNFRSF1A levels (pg/ml). The normal range is $746-1966 \mathrm{pg} / \mathrm{ml}$.

level in the affected father was towards the lower limit of normal $(978 \mathrm{pg} / \mathrm{ml}$ ) (normal range $746-1966 \mathrm{pg} / \mathrm{ml}$ ). Soluble TNFRSF1B levels were normal in all family members.

\section{Discussion}

Recent developments in the molecular genetics of periodic fevers have facilitated the diagnoses of these patients. The novel R92P mutation found in two unaffected carriers suggests that this mutation is less penetrant and further supports the concept that even in 'simple' Mendelian disorders the disease phenotypes are, in fact, complex traits. ${ }^{8}$ Among the possible modifiers of the TRAPS phenotype in this family include gene environment interactions, background genes, and varying thresholds for modifier protein function. Some possible background genes that may be involved include metalloprotease cleavage enzymes involved in TNF and TNF receptor shedding, in addition to disintegrin docking molecules which interact with these enzymes. The approximately half normal plasma TNFRSF1A levels found in the two unaffected carriers aged 35 and 38 years respectively, plus one mildly affected individual, compared with the normal levels in the affected father may reflect the compensatory increase in TNFRSF1A levels found during attacks of fever in the more severely affected members. It is also notable that the low plasma TNFRSF1A levels segregate completely with the mutation in all the children, including those who are unaffected.

Other cases of non-penetrant TNFRSF1A mutations have been described; ${ }^{1}$ some members of the original T50M Irish family have mild symptoms in comparison with the severely affected proband, and one woman with this mutation remains asymptomatic in her mid-50s. Likewise, one male member of the Finnish family with the C88Y mutation is asymptomatic in his early 20 s. It is notable that all these unaffected individuals have low plasma levels of soluble TNFRSF1A, as is the case with the asymptomatic members of this Dutch family and it is possible that low levels of soluble TNFRSF1A in combination with particular environmental insults may be necessary to produce the fullblown TRAPS phenotype.

There was no genetic evidence to support a diagnosis of either FMF or HIDS in affected members of thisfamily in view of the dominant pattern of inheritance (parent-child transmission) and lack of haplotype sharing in the offspring. Furthermore, the clinical presentation (long duration of attacks and no lymphadenopathy) did not fit the clinical picture of either FMF or HIDS. 


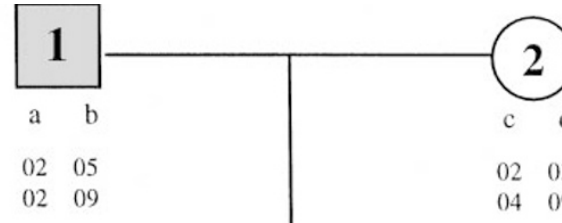

HIDS locus

markers

D12S306

D12S79
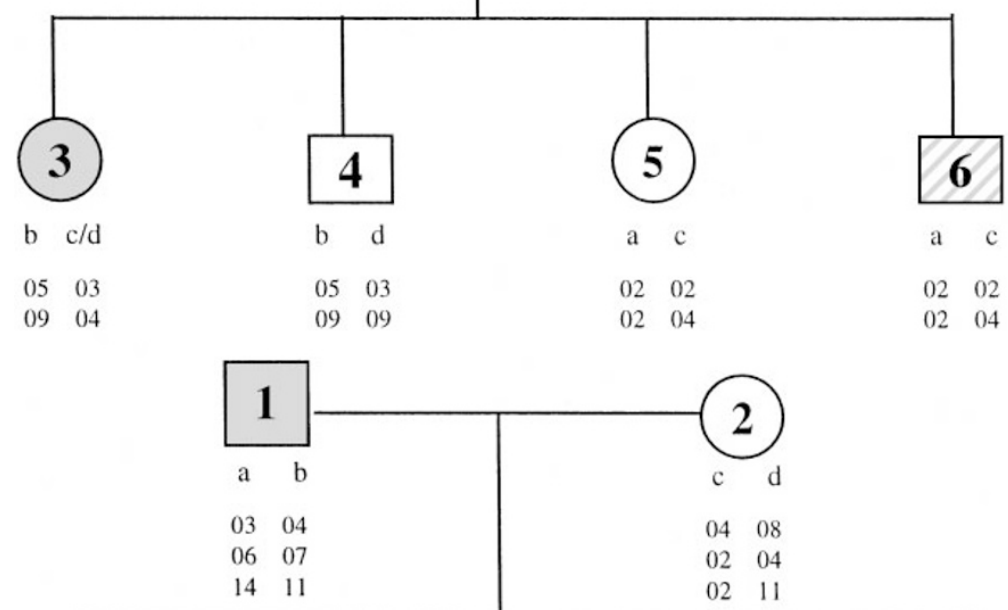

FMF locus

markers

D16S3070

$04 \quad 08$

D $16 \$ 3275$

$02 \quad 11$

D16S418
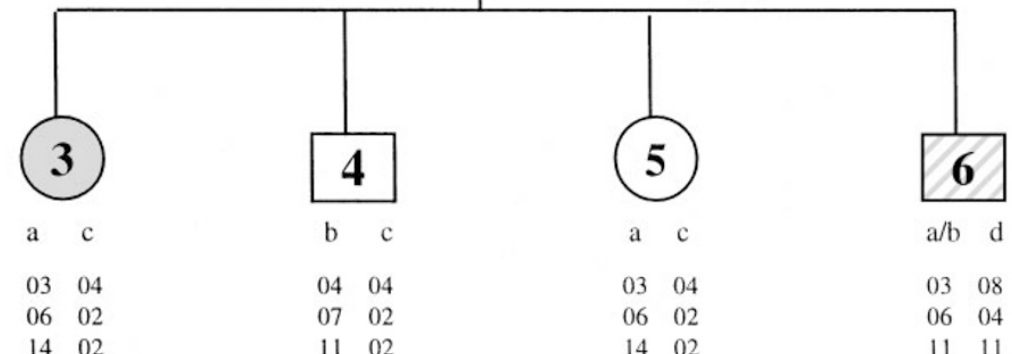

a c

a/b d

$03 \quad 04$

$06 \quad 02$

$03 \quad 08$

1402

$06 \quad 04$

1111

Figure 2 The Dutch pedigree showing markers spanning the HIDS and FMF loci; there is no evidence of haplotype sharing in subjects 3 and 6 .

This result also raises the question as to whether TNFRSF1A mutations may be present in mildly symptomatic or indeed asymptomatic members of the overall community. In this and previous studies we have not found mutations in unaffected controls but clearly a larger study is required to exclude formally this possibility.

\section{Acknowledgements}

We would like to express our gratitude to the family for agreeing to participate in the study. This work was supported by the Research Advisory Committee of the Special Trustees of St Bartholomew's and the Royal London Hospitals NHS Trust.

\section{References}

1 McDermott MF, Aksentijevich I, Galon J et al: Germline mutations in the extracellular domains of the $55 \mathrm{kDa}$ TNF receptor (TNF-R1) define a family of dominantly inherited autoinflammatory syndromes. Cell 1999; 97: 133-144.

2 McDermott MF: Autosomal dominant recurrent fevers - clinical and genetic aspects. Rev Rhum Engl Ed. 1999; 66: 484-491.
3 Williamson LM, Hull D, Mehta R, Reeves WG, Robinson BHB, Toghill PH: Familial hibernian fever. Quart J Med 1982; 204: 469-480.

4 Kastner DL: Intermittent and periodic arthritis syndromes. In Koopman W (ed). Arthritis and Allied Conditions: a Textbook of Rheumatology, Williams \& Wilkins: Baltimore, 1997, 13th edn, pp 1279-1306.

5 Zweers EJ, Erkelens DW: Een Nederlandse familie met familiale mediterrane koorts. (A Dutch family with familial Mediterranean fever). Ned Tijdschr Geneeskd 1993; 137: 1570-1573.

6 Eskdale J, Turestkaya RL, Armstrong C, Kuprash DV, Nedospasov SA, Gallagher G: A polymorphic microsatellite marker in the human p55 receptor, CD120a. Genes Immun 2000; 1: 228-230.

7 Drenth JPH, Cuisset L, Grateau G, Vasseur C, van de Velde-Visser $\mathrm{SD}$, de Jong JGN and contributing members of the International Hyper-IgD Study Group: Mutations in the gene encoding mevalonate cause hyper-IgD and periodic fever syndrome. Nat Genet 1999; 22: 178-181.

8 Dipple KM, McCabe ERB: Phenotypes of patients with 'simple' Mendelian disorders are complex traits: thresholds, modifiers, and systems dynamics. Am J Hum Genet 2000; 66: 1729-1735. 